\title{
The Global Weak Solution for a Generalized Camassa-Holm Equation
}

\author{
Shaoyong Lai \\ Department of Applied Mathematics, Southwestern University of Finance and Economics, Chengdu, Sichuan 610074, China
}

Correspondence should be addressed to Shaoyong Lai; laishaoy@swufe.edu.cn

Received 25 October 2012; Accepted 24 December 2012

Academic Editor: Yong Hong Wu

Copyright (C) 2013 Shaoyong Lai. This is an open access article distributed under the Creative Commons Attribution License, which permits unrestricted use, distribution, and reproduction in any medium, provided the original work is properly cited.

A nonlinear generalization of the famous Camassa-Holm model is investigated. Provided that initial value $u_{0} \in H^{s}(R)(1 \leq s \leq 3 / 2)$ and $\left(1-\partial_{x}^{2}\right) u_{0}$ satisfies an associated sign condition, it is shown that there exists a unique global weak solution to the equation in space $u(t, x) \in L^{2}\left([0,+\infty), H^{s}(R)\right)$ in the sense of distribution, and $u_{x} \in L^{\infty}([0,+\infty) \times R)$.

\section{Introduction}

In recent years, a lot of works have been carried out to investigate the Camassa-Holm equation [1],

$$
u_{t}-u_{t x x}+k u_{x}+3 u u_{x}=2 u_{x} u_{x x}+u u_{x x x}
$$

which is a completely integrable equation. In fact, the Camassa-Holm equation arises as a model describing the unidirectional propagation of shallow water waves over a flat bottom [1-3]. The equation was originally derived much earlier as a bi-Hamiltonian generalization of the Kortewegde Vries equation (see [4]). Johnson [2], Constantin and Lannes [5] derived models which include the Camassa-Holm equation (1). It has been found that (1) conforms with many conservation laws (see $[6,7]$ ) and possesses smooth solitary wave solutions if $k>0[3,8]$ or peakons if $k=0[3,9]$. Equation (1) is also regarded as a model of the geodesic flow for the $H^{1}$ right invariant metric on the Bott-Virasoro group if $k>0$ and on the diffeomorphism group if $k=0$ (see [10-14]). The well-posedness of local strong solutions for generalized forms of (1) has been given in [15-17]. The sharpest results for the global existence and blow-up solutions are found in Bressan and Constantin $[18,19]$.

Recently, Li et al. [20] studied the following generalized Camassa-Holm equation:

$$
\begin{array}{r}
u_{t}-u_{t x x}+k u^{m} u_{x}+(m+3) u^{m+1} u_{x} \\
=(m+2) u^{m} u_{x} u_{x x}+u^{m+1} u_{x x x},
\end{array}
$$

where $m \geq 0$ is a natural number. Obviously, (2) reduces to (1) if $m=0$. The authors applied the pseudoparabolic regularization technique to build the local well-posedness for (2) in Sobolev space $H^{s}(R)$ with $s>3 / 2$ via a limiting procedure. Provided that the initial value $u_{0}$ satisfies a sign condition and $u_{0} \in H^{s}(R)(s>3 / 2)$, it is shown that there exists a unique global strong solution for (2) in space $C\left([0, \infty) ; H^{s}(R)\right) \cap C^{1}\left([0, \infty) ; H^{s-1}(R)\right)$. However, the existence and uniqueness of the global weak solution for (2) is not investigated in [20].

The objective of this paper is to establish the wellposedness of global weak solutions for (2). Using the estimates in $H^{q}(R)$ with $0 \leq q \leq 1 / 2$, which are derived from the equation itself, we prove that there exists a unique global weak solution to (2) in space $H^{s}(R)$ with $1 \leq s \leq 3 / 2$ if $u_{0} \in H^{s}(R)$, and $\left(1-\partial_{x}^{2}\right) u_{0}$ satisfies an associated sign condition.

The structure of this paper is as follows. The main result is given in Section 2. Several lemmas are given in Section 3. Section 4 establishes the proof of the main result.

\section{Main Results}

Firstly, we give some notations.

The space of all infinitely differentiable functions $\phi(t, x)$ with compact support in $[0,+\infty) \times R$ is denoted by $C_{0}^{\infty}$. $L^{p}=L^{p}(R)(1 \leq p<+\infty)$ is the space of all measurable functions $h$ such that $\|h\|_{L^{p}}^{p}=\int_{R}|h(t, x)|^{p} d x<$ $\infty$. We define $L^{\infty}=L^{\infty}(R)$ with the standard norm 
$\|h\|_{L^{\infty}}=\inf _{m(e)=0} \sup _{x \in R \backslash e}|h(t, x)|$. For any real number $s$, we let $H^{s}=H^{s}(R)$ denote the Sobolev space with the norm defined by

$$
\|h\|_{H^{s}}=\left(\int_{R}\left(1+|\xi|^{2}\right)^{s}|\widehat{h}(t, \xi)|^{2} d \xi\right)^{1 / 2}<\infty
$$

where $\widehat{h}(t, \xi)=\int_{R} e^{-i x \xi} h(t, x) d x$.

For $T>0$ and nonnegative number $s$, let $C\left([0, T) ; H^{s}(R)\right)$ denote the Frechet space of all continuous $H^{s}$-valued functions on $[0, T)$. We set $\Lambda=\left(1-\partial_{x}^{2}\right)^{1 / 2}$.

Defining

$$
\phi(x)= \begin{cases}e^{1 /\left(x^{2}-1\right)}, & |x|<1 \\ 0, & |x| \geq 1\end{cases}
$$

and letting $\phi_{\varepsilon}(x)=\varepsilon^{-(1 / 4)} \phi\left(\varepsilon^{-(1 / 4)} x\right)$ with $0<\varepsilon<1 / 4$ and $u_{\varepsilon 0}=\phi_{\varepsilon} \star u_{0}$ (convolution of $\phi_{\varepsilon}$ and $u_{0}$ ), we know that $u_{\varepsilon 0} \in$ $C^{\infty}$ for any $u_{0} \in H^{s}$ with $s>0$. Notation $\left(1-\partial_{x}^{2}\right) u+k / 2(m+$ $1) \in N^{+}(R)$ (or equivalently $\left.\left(1-\partial_{x}^{2}\right) u+k / 2(m+1) \in N^{-}(R)\right)$ means that $\left(1-\partial_{x}^{2}\right) u \star \phi_{\varepsilon}+k / 2(m+1) \geq 0$ (or equivalently $\left.\left(1-\partial_{x}^{2}\right) u \star \phi_{\varepsilon}+k / 2(m+1) \leq 0\right)$ for an arbitrary sufficiently small $\varepsilon>0$.

For the equivalent form of (2), we consider its Cauchy problem

$$
\begin{aligned}
& u_{t}-u_{t x x}=-\frac{k}{m+1}\left(u^{m+1}\right)_{x}-\frac{m+3}{m+2}\left(u^{m+2}\right)_{x} \\
&+\frac{1}{m+2} \partial_{x}^{3}\left(u^{m+2}\right)-(m+1) \partial_{x}\left(u^{m} u_{x}^{2}\right) \\
&+u^{m} u_{x} u_{x x} \\
& u(0, x)=u_{0}(x) .
\end{aligned}
$$

Definition 1. A function $u(t, x) \in L^{2}\left([0,+\infty), H^{s}(R)\right)$ is called a global weak solution to problem (5) if for every $T>0$, $u(t, x) \in H^{s}(R), u_{t}(t, x) \in H^{s-1}(R)$, and all $\psi(t, x) \in C_{0}^{\infty}$, it holds that

$$
\begin{aligned}
& \int_{0}^{T} \int_{R}\left[u_{t}-u_{t x x}+k u^{m} u_{x}+(m+3) u^{m+1} u_{x}\right. \\
& \left.\quad-(m+2) u^{m} u_{x} u_{x x}-u^{m+1} u_{x x x}\right] \psi(t, \mathrm{x}) d x d t=0
\end{aligned}
$$

with $u(0, x)=u_{0}(x)$.

Now, we give the main result of this work.

Theorem 2. Let $u_{0}(x) \in H^{s}(R), 1 \leq s \leq 3 / 2,\left(1-\partial_{x}^{2}\right) u_{0}+$ $k / 2(m+1) \in N^{+}(R)$, and $k \geq 0$ (or equivalently $\left(1-\partial_{x}^{2}\right) u_{0}+$ $\left.k / 2(m+1) \in N^{-}(R), k \leq 0\right)$. Then, problem (5) has a unique global weak solution $u(t, x) \in L^{2}\left([0,+\infty), H^{s}(R)\right)$ in the sense of distribution, and $u_{x} \in L^{\infty}([0,+\infty) \times R)$.

\section{Several Lemmas}

Lemma 3 (see [20]). Let $u_{0}(x) \in H^{s}(R)$ with $s>3 / 2$. Then, the Cauchy problem (5) has a unique solution

$$
u(t, x) \in C\left([0, T) ; H^{s}(R)\right) \bigcap C^{1}\left([0, T) ; H^{s-1}(R)\right),
$$

where $T>0$ depends on $\left\|u_{0}\right\|_{H^{s}(R)}$.

Lemma 4 (see [20]). Let $u_{0}(x) \in H^{s}, s>3 / 2$, and $k \geq$ $0,\left(1-\partial_{x}^{2}\right) u_{0}+k / 2(m+1) \geq 0$ (or equivalently $k \leq 0,(1-$ $\left.\left.\partial_{x}^{2}\right) u_{0}+k / 2(m+1) \leq 0\right)$. Then, problem (5) has a unique solution satisfying

$$
u(t, x) \in C\left([0, \infty) ; H^{s}(R)\right) \bigcap C^{1}\left([0, \infty) ; H^{s-1}(R)\right) .
$$

Using the first equation of system (5) derives

$$
\frac{d}{d t} \int_{R}\left(u^{2}+u_{x}^{2}\right) d x=0
$$

from which one has the conservation law

$$
\int_{R}\left(u^{2}+u_{x}^{2}\right) d x=\int_{R}\left(u_{0}^{2}+u_{0 x}^{2}\right) d x .
$$

Lemma 5 (see [20]). Let $s>3 / 2$, and the function $u(t, x)$ is a solution of problem (5) and the initial data $u_{0}(x) \in H^{s}$. Then, the following inequality holds:

$$
\|u\|_{H^{1}}^{2} \leq \int_{R}\left(u^{2}+u_{x}^{2}\right) d x=\int_{R}\left(u_{0}^{2}+u_{0 x}^{2}\right) d x .
$$

For $q \in(0, s-1]$, there is a constant $c$ such that

$$
\begin{aligned}
\int_{R}\left(\Lambda^{q+1} u\right)^{2} d x \leq & \int_{R}\left(\Lambda^{q+1} u_{0}\right)^{2} d x \\
& +c \int_{0}^{t}\|u\|_{H^{q+1}}^{2}\left(\left\|u_{x}\right\|_{L^{\infty}}\|u\|_{L^{\infty}}^{m}\right. \\
& \left.+\|u\|_{L^{\infty}}^{m-1}\left\|u_{x}\right\|_{L^{\infty}}^{2}\right) d \tau .
\end{aligned}
$$

For $q \in[0, s-1]$, there is a constant $c$ such that

$$
\begin{gathered}
\left\|u_{t}\right\|_{H^{q}} \leq c\|u\|_{H^{q+1}}\left(\|u\|_{L^{\infty}}^{m}\|u\|_{H^{1}}+\|u\|_{L^{\infty}}^{m}\left\|u_{x}\right\|_{L^{\infty}}\right. \\
\left.+\|u\|_{L^{\infty}}^{m-1}\left\|u_{x}\right\|_{L^{\infty}}^{2}\right) .
\end{gathered}
$$

For (2), consider the problem

$$
\begin{gathered}
p_{t}=u^{m+1}(t, p), \quad t \in[0, T), \\
p(0, x)=x .
\end{gathered}
$$

Lemma 6 (see [20]). Let $u_{0} \in H^{s}, s \geq 3$, and let $T>0$ be the maximal existence time of the solution to problem (5). Then, problem (14) has a unique solution $p \in C^{1}([0, T) \times R)$. Moreover, the map $p(t, \cdot)$ is an increasing diffeomorphism of $R$ with $p_{x}(t, x)>0$ for $(t, x) \in[0, T) \times R$. 
Differentiating (14) with respect to $x$ yields

$$
\begin{gathered}
\frac{d}{d t} p_{x}=(m+1) u^{m} u_{x}(t, p) p_{x}, \quad t \in[0, T), \\
p_{x}(0, x)=1,
\end{gathered}
$$

which leads to

$$
p_{x}(t, x)=\exp \left(\int_{0}^{t}(m+1) u^{m} u_{x}(\tau, p(\tau, x)) d \tau\right) .
$$

The next lemma is reminiscent of a strong invariance property of the Camassa-Holm equation (the conservation of momentum [21]).

Lemma 7 (see [20]). Let $u_{0} \in H^{s}$ with $s \geq 3$, and let $T>0$ be the maximal existence time of the problem (5). It holds that

$$
y(t, p(t, x)) p_{x}^{2}(t, x)=y_{0}(x) e^{\int_{0}^{t} m u^{m} u_{x} d \tau}
$$

where $(t, x) \in[0, T) \times R$ and $y:=u-u_{x x}+k / 2(m+1)$.

Lemma 8. If $u_{0} \in H^{s}, s \geq 3$, such that $\left(1-\partial_{x}^{2}\right) u_{0}+k / 2(m+1) \geq$ $0, k \geq 0$ (or equivalently, $\left(1-\partial_{x}^{2}\right) u_{0}+k / 2(m+1) \leq 0, k \leq 0$ ), then the solution of problem (5) satisfies

$$
\left\|u_{x}\right\|_{L^{\infty}} \leq\|u\|_{L^{\infty}}+\frac{|k|}{2(m+1)} \leq c .
$$

Proof. Using $u_{0}-u_{0 x x}+k / 2(m+1) \geq 0$, it follows from Lemma 7 that $u-u_{x x}+k / 2(m+1) \geq 0$. Letting $Y_{1}=u-u_{x x}$, we have

$$
u=\frac{1}{2} e^{-x} \int_{-\infty}^{x} e^{\eta} Y_{1}(t, \eta) d \eta+\frac{1}{2} e^{x} \int_{x}^{\infty} e^{-\eta} Y_{1}(t, \eta) d \eta
$$

from which we obtain

$$
\begin{aligned}
\partial_{x} u(t, x) & -\frac{1}{2}\left(e^{-x} \int_{-\infty}^{x} e^{\eta} Y_{1}(t, \eta) d \eta+e^{x} \int_{x}^{\infty} e^{-\eta} Y_{1}(t, \eta) d \eta\right) \\
& +e^{x} \int_{x}^{\infty} e^{-\eta} Y_{1}(t, \eta) d \eta \\
= & -u(t, x)+e^{x} \int_{x}^{\infty} e^{-\eta} Y_{1}(t, \eta) d \eta \\
= & -u(t, x)+e^{x} \int_{x}^{\infty} e^{-\eta}\left(Y_{1}(t, \eta)+\frac{k}{2(m+1)}\right) d \eta \\
& -\frac{k}{2(m+1)} e^{x} \int_{x}^{\infty} e^{-\eta} d \eta \\
= & -u(t, x)+e^{x} \int_{x}^{\infty} e^{-\eta}(y(t, \eta)) d \eta-\frac{k}{2(m+1)} \\
\geq & -u(t, x)-\frac{k}{2(m+1)} .
\end{aligned}
$$

On the other hand, we have

$$
\begin{aligned}
& \partial_{x} u(t, x) \\
&=\frac{1}{2}\left(e^{-x} \int_{-\infty}^{x} e^{\eta} Y_{1}(t, \eta) d \eta+e^{x} \int_{x}^{\infty} e^{-\eta} Y_{1}(t, \eta) d \eta\right) \\
&-e^{-x} \int_{-\infty}^{x} e^{\eta} Y_{1}(t, \eta) d \eta \\
&= u(t, x)-e^{-x} \int_{-\infty}^{x} e^{\eta} Y_{1}(t, \eta) d \eta \\
&= u(t, x)-e^{-x} \int_{-\infty}^{x} e^{\eta}\left(Y_{1}(t, \eta)+\frac{k}{2(m+1)}\right) d \eta \\
&+\frac{k}{2(m+1)} e^{-x} \int_{-\infty}^{x} e^{\eta} d \eta \\
&= u(t, x)-e^{-x} \int_{-\infty}^{x} e^{\eta} y(t, \eta) d \eta+\frac{k}{2(m+1)} \\
& \leq u(t, x)+\frac{k}{2(m+1)} .
\end{aligned}
$$

The inequalities (19), (20), and (21) derive that inequality (18) is valid. Similarly, if $\left(1-\partial_{x}^{2}\right) u_{0}+k / 2(m+1) \leq 0, k \leq 0$, we still know that (18) is valid.

Lemma 9. For $s>0, u_{0} \in H^{s}$, it holds that

$$
\begin{gathered}
\left\|u_{\varepsilon 0 x}\right\|_{L^{\infty}} \leq c\left\|u_{0 x}\right\|_{L^{\infty}}, \\
\left\|u_{\varepsilon 0}\right\|_{H^{q}} \leq c, \quad \text { if } q \leq s, \\
\left\|u_{\varepsilon 0}\right\|_{H^{q}} \leq c \varepsilon^{s-q / 4}, \quad \text { if } q>s, \\
\left\|u_{\varepsilon 0}-u_{0}\right\|_{H^{q}} \leq c \varepsilon^{s-q / 4}, \quad \text { if } q \leq s, \\
\left\|u_{\varepsilon 0}-u_{0}\right\|_{H^{s}}=o(1),
\end{gathered}
$$

where $c$ is a constant independent of $\varepsilon$.

The proof of this lemma can be found in Lai and $\mathrm{Wu}$ [15]. From Lemma 3, it derives that the Cauchy problem

$$
\begin{aligned}
u_{t}-u_{t x x}= & -\frac{m+3}{m+2}\left(u^{m+2}\right)_{x}+\frac{1}{m+2} \partial_{x}^{3}\left(u^{m+2}\right) \\
& -(m+1) \partial_{x}\left(u^{m} u_{x}^{2}\right)+u^{m} u_{x} u_{x x} \\
u & (0, x)=u_{\varepsilon 0}(x), \quad x \in R
\end{aligned}
$$

has a unique solution $u$ depending on the parameter $\varepsilon$. We write $u_{\varepsilon}(t, x)$ to represent the solution of problem (23). Using Lemma 3 derives that $u_{\varepsilon}(t, x) \in C^{\infty}\left([0, T), H^{\infty}(R)\right)$ since $u_{\varepsilon 0}(x) \in C_{0}^{\infty}(R)$. 
Lemma 10. Provided that $u_{0} \in H^{s}, 1 \leq s \leq 3 / 2, k \geq 0$, and $\left(1-\partial_{x}^{2}\right) u_{0}+k / 2(m+1) \in N^{+}(R)$ (or equivalently $\left(1-\partial_{x}^{2}\right) u_{0}+$ $\left.k / 2(m+1) \in N^{-}(R), k \leq 0\right)$, then there exists a constant $c_{0}>0$ independent of $\varepsilon$ such that the solution of problem (23) satisfies

$$
\left\|u_{\varepsilon x}\right\|_{L^{\infty}} \leq\left\|u_{x}\right\|_{L^{\infty}}+\frac{|k|}{2(m+1)} \leq c_{0} .
$$

Proof. Using identity (10) and Lemma 9, if $u_{0} \in H^{S}(R)$ with $1 \leq s \leq 3 / 2$, we have

$$
\left\|u_{\varepsilon}\right\|_{L^{\infty}} \leq\left\|u_{\varepsilon}\right\|_{H^{1}}=\left\|u_{\varepsilon 0}\right\|_{H^{1}} \leq c
$$

where $c$ is independent of $\varepsilon$.

From Lemma 8, we have

$$
\left\|u_{\varepsilon x}\right\|_{L^{\infty}} \leq\left\|u_{\varepsilon}\right\|_{L^{\infty}}+\frac{|k|}{2(m+1)} \leq c+\frac{|k|}{2(m+1)},
$$

which completes the proof.

Lemma 11. For any $f_{1} \in L^{\infty}, f_{2} \in H^{z}$ with $z \leq 0$, it holds that

$$
\left\|f_{1} f_{2}\right\|_{H^{z}} \leq c\left\|f_{1}\right\|_{L^{\infty}}\left\|f_{2}\right\|_{H^{z}} \text { for any } z \leq 0 \text {. }
$$

The proof of this lemma can be found in [15].

\section{Existence and Uniqueness of Global Weak Solution}

Provided that $1 \leq s \leq 3 / 2$, for problem (23), applying Lemmas 5, 9, and 10, and the Gronwall's inequality, we obtain the inequalities

$$
\begin{gathered}
\left\|u_{\varepsilon}\right\|_{H^{1}} \leq\left\|u_{\varepsilon 0}\right\|_{H^{1}} \leq c, \\
\left\|u_{\varepsilon}\right\|_{H^{q}} \leq c\left\|u_{\varepsilon 0}\right\|_{H^{q}} \exp \left[\int_{0}^{t}\left(\left\|u_{\varepsilon x}\right\|+\left\|u_{\varepsilon x}\right\|_{L^{\infty}}^{2}\right) d \tau\right] \leq c e^{c t}, \\
\left\|u_{\varepsilon t}\right\|_{H^{r}} \leq\left\|u_{\varepsilon}\right\|_{H^{r+1}}\left(1+e^{c t}\right) \leq c\left(1+e^{c t}\right),
\end{gathered}
$$

where $q \in(0, s], r \in[0, s-1]$, and $c$ is a constant independent of $\varepsilon$. It follows from the Aubin's compactness theorem that there is a subsequence of $\left\{u_{\varepsilon}\right\}$, denoted by $\left\{u_{\varepsilon_{p}}\right\}$, such that $\left\{u_{\varepsilon_{n}}\right\}$ and their temporal derivatives $\left\{u_{\varepsilon_{n}}\right\}$ are weakly convergent to a function $u(t, x)$ and its derivative $u_{t}$ in $L^{2}\left([0, T], H^{s}\right)$ and $L^{2}\left([0, T], H^{s-1}\right)$, respectively, where $T$ is an arbitrary fixed positive number. Moreover, for any real number $R_{1}>0$, $\left\{u_{\varepsilon_{n}}\right\}$ is convergent to the function $u$ strongly in the space
$L^{2}\left([0, T], H^{q}\left(-R_{1}, R_{1}\right)\right)$ for $q \in(0, s]$ and $\left\{u_{\varepsilon_{n} t}\right\}$ converges to $u_{t}$ strongly in the space $L^{2}\left([0, T], H^{r}\left(-R_{1}, R_{1}\right)\right)$ for $r \in$ $[0, s-1]$.

4.1. The Proof of Existence for Global Weak Solution. For an arbitrary fixed $T>0$, from Lemma 10, we know that $\left\{u_{\varepsilon_{n} x}\right\}\left(\varepsilon_{n} \rightarrow 0\right)$ is bounded in the space $L^{\infty}$. Thus, the sequences $\left\{u_{\varepsilon_{n}}\right\},\left\{u_{\varepsilon_{n} x}\right\},\left\{u_{\varepsilon_{n} x}^{2}\right\}$, and $\left\{u_{\varepsilon_{n} x}^{3}\right\}$ are weakly convergent to $u, u_{x}, u_{x}^{2}$, and $u_{x}^{3}$ in $L^{2}\left([0, T], H^{r}\left(-R_{1}, R_{1}\right)\right)$ for any $r \in[0, s-1)$, separately. Using $u^{m}\left(u_{x}^{2}\right)_{x}=\left(u^{m} u_{x}^{2}\right)_{x}-\left(u^{m}\right)_{x} u_{x}^{2}$, we know that $u$ satisfies the equation

$$
\begin{gathered}
-\int_{0}^{T} \int_{R} u\left(g_{t}-g_{x x t}\right) d x d t \\
=\int_{0}^{T} \int_{R}\left[\left(\frac{m+3}{m+2} u^{m+2}+(m+1) u^{m} u_{x}^{2}\right) g_{x}\right. \\
-\frac{1}{m+2} u^{m+2} g_{x x x}-\frac{1}{2} u^{m} u_{x}^{2} g_{x} \\
\left.-\frac{m}{2} u^{m-1} u_{x}^{3} g\right] d x d t
\end{gathered}
$$

with $u(0, x)=u_{0}(x)$ and $g \in C_{0}^{\infty}$. Since $X=L^{1}([0, T] \times R)$ is a separable Banach space and $\left\{u_{\varepsilon_{n} x}\right\}$ is a bounded sequence in the dual space $X^{*}=L^{\infty}([0, T] \times R)$ of $X$, there exists a subsequence of $\left\{u_{\varepsilon_{n} x}\right\}$, still denoted by $\left\{u_{\varepsilon_{n} x}\right\}$, weakly star convergent to a function $v$ in $L^{\infty}([0, T] \times R)$. As $\left\{u_{\varepsilon_{n} x}\right\}$ weakly converges to $u_{x}$ in $L^{2}([0, T] \times R)$, it results that $u_{x}=v$ almost everywhere. Thus, we obtain $u_{x} \in L^{\infty}([0, T] \times R)$. Since $\mathrm{T}>0$ is an arbitrary number, we complete the global existence of weak solutions to problem (5).

Proof of Uniqueness. Suppose that there exist two global weak solutions $u(t, x)$ and $v(t, x)$ to problem (5) with the same initial value $u(0, x) \in H^{s}(R), 1 \leq s \leq 3 / 2$, we consider its associated regularized problem (23). Letting $w_{\varepsilon}=$ $u_{\varepsilon}(t, x)-v_{\varepsilon}(t, x)$, from Lemma 10 , we get $\left\|\partial u_{\varepsilon(t, x)} / \partial x\right\|_{L^{\infty}} \leq$ $c$ and $\left\|\partial v_{\varepsilon(t, x)} / \partial x\right\|_{L^{\infty}} \leq c$ which is independent of $\varepsilon$. Still denoting $u=u_{\varepsilon}, v=v_{\varepsilon}$, and $w=w_{\varepsilon}$, it holds that

$$
\begin{gathered}
w_{t}=\left(1-\partial_{x}^{2}\right)^{-1}\left[-\partial_{x}\left(u^{m+2}-v^{m+2}\right)\right. \\
-\partial_{x}\left[\partial_{x}\left(u^{m+1}\right) \partial_{x} w\right. \\
\left.+\partial_{x}\left(u^{m+1}-v^{m+1}\right) \partial_{x} v\right] \\
\left.+\left[u^{m} u_{x} u_{x x}-v^{m} v_{x} v_{x x}\right]\right] \\
-\frac{1}{m+2} \partial_{x}\left(u^{m+2}-v^{m+2}\right), \\
w(0, x)=0 .
\end{gathered}
$$


Multiplying both sides of (30) by $w$, we get

$$
\begin{aligned}
\frac{1}{2} \frac{d}{d t} \int_{R} w^{2} d x \leq c & \left|\int_{R} w\left(u^{m+2}-v^{m+2}\right)_{x} d x\right| \\
& +\left|\int_{R} w \Lambda^{-2}\left(u^{m+2}-v^{m+2}\right)_{x} d x\right| \\
& +\left|\int_{R} w \Lambda^{-2}\left[\partial_{x}\left(u^{m+1}\right) \partial_{x} w\right]_{x} d x\right| \\
& +\left|\int_{R} w \Lambda^{-2}\left[\partial_{x}\left(u^{m+1}-v^{m+1}\right) \partial_{x} v\right]_{x} d x\right| \\
& +\left|\int_{R} w \Lambda^{-2}\left[u^{m} u_{x} u_{x x}-v^{m} v_{x} v_{x x}\right] d x\right| \\
= & I_{1}+I_{2}+I_{3}+I_{4}+I_{5} .
\end{aligned}
$$

Using $\|u\|_{L^{\infty}} \leq c,\|v\|_{L^{\infty}} \leq c,\left\|u_{x}\right\|_{L^{\infty}} \leq c,\left\|v_{x}\right\|_{L^{\infty}} \leq c$, we have

$$
\begin{aligned}
& I_{1} \leq c\left|\int_{R} w\left[w \sum_{j=0}^{m+1} u^{j} v^{m+1-j}\right]_{x} d x\right| \\
& =c\left|\int_{R} w\left[w_{x} \sum_{j=0}^{m+1} u^{j} v^{m+1-j}+w \sum_{j=0}^{m+1}\left(u^{j} v^{m+1-j}\right)_{x}\right] d x\right| \\
& =c\left|\int_{R}\left(\frac{1}{2} w^{2}\right)_{x} \sum_{j=0}^{m+1} u^{j} v^{m+1-j}+w^{2} \sum_{j=0}^{m+1}\left(u^{j} v^{m+1-j}\right)_{x} d x\right| \\
& =c\left|\int_{R}\left(\frac{-1}{2} w^{2}\right) \sum_{j=0}^{m+1}\left(u^{j} v^{m+1-j}\right)_{x}+w^{2} \sum_{j=0}^{m+1}\left(u^{j} v^{m+1-j}\right)_{x} d x\right| \\
& =c\left|\int_{R}\left(\frac{1}{2} \mathrm{w}^{2}\right) \sum_{j=0}^{m+1}\left(u^{j} v^{m+1-j}\right)_{x} d x\right| \\
& \leq c\|w\|_{L^{2}}^{2} \sum_{j=0}^{m+1}\left\|\left(u^{j} v^{m+1-j}\right)_{x}\right\|_{L^{\infty}} \\
& \leq c\|w\|_{L^{2}}^{2} .
\end{aligned}
$$

Applying Lemma 11 repeatedly, we have

$$
\begin{aligned}
I_{2} & \leq c\|w\|_{L^{2}}\left\|\Lambda^{-2}\left(u^{m+2}-v^{m+2}\right)_{x}\right\|_{L^{2}} \\
& \leq c\|w\|_{L^{2}}\left\|w \sum_{j=0}^{m+1} u^{j} v^{m+1-j}\right\|_{L^{2}} \\
& \leq c\|w\|_{L^{2}}^{2} \sum_{j=0}^{m+1}\|u\|_{L^{\infty}}^{j}\|v\|_{L^{\infty}}^{m+1-j} \\
& \leq c\|w\|_{L^{2}}^{2},
\end{aligned}
$$

$$
\begin{aligned}
I_{3} & \leq c\|w\|_{L^{2}}\left\|\Lambda^{-2}\left[\partial_{x}\left(u^{m+1}\right) \partial_{x} w\right]_{x}\right\|_{L^{2}} \\
& \leq c\|w\|_{L^{2}}\left\|\partial_{x}\left(u^{m+1}\right) \partial_{x} w\right\|_{H^{-1}} \\
& \leq c\|w\|_{L^{2}}\left\|\partial_{x} w\right\|_{H^{-1}}\left\|\partial_{x}\left(u^{m+1}\right)\right\|_{L^{\infty}} \\
& \leq c\|w\|_{L^{2}}^{2}, \\
I_{4} & \leq c\|w\|_{L^{2}}\left\|\partial_{x}\left(u^{m+1}-v^{m+1}\right) \partial_{x} v\right\|_{H^{-1}} \\
& \leq c\|w\|_{L^{2}}\left\|\partial_{x} v\right\|_{L^{\infty}}\left\|\partial_{x}\left(u^{m+1}-v^{m+1}\right)\right\|_{H^{-1}} \\
& \leq c\|w\|_{L^{2}}\left\|u^{m+1}-v^{m+1}\right\| H_{H^{0}} \\
& \leq c\|w\|_{L^{2}}\left\|w \sum_{j=0}^{m} u^{j} v^{m-j}\right\|_{L^{2}} \\
& \leq c\|w\|_{L^{2}}^{2} \sum_{j=0}^{m}\|u\|_{L^{\infty}}^{j}\|v\|_{L^{\infty}}^{m-j} \\
& \leq c\|w\|_{L^{2}}^{2} .
\end{aligned}
$$

For $I_{5}$, using Lemma 11 derives

$$
\begin{aligned}
& I_{5} \leq c\|w\|_{L^{2}}\left\|\left(u^{m}-v^{m}\right)\left(u_{x}^{2}\right)_{x}+v^{m}\left[u_{x}^{2}-v_{x}^{2}\right]_{x}\right\|_{H^{-2}} \\
& \leq c\|w\|_{L^{2}}\left\|\left(u^{m}-v^{m}\right)\left(u_{x}^{2}\right)_{x}\right\|_{H^{-2}}+\left\|v^{m}\left[u_{x}^{2}-v_{x}^{2}\right]_{x}\right\|_{H^{-2}} \\
& \leq c\|w\|_{L^{2}}\left(\left\|\left[\left(u^{m}-v^{m}\right)\left(u_{x}^{2}\right)\right]_{x}-\left(u^{m}-v^{m}\right)_{x} u_{x}^{2}\right\|_{H^{-2}}\right. \\
& \left.\quad+\|v\|_{L^{\infty}}^{m}\left\|(u-v)_{x}\left(u_{x}+v_{x}\right)\right\|_{H^{-1}}\right) \\
& \leq c\|w\|_{L^{2}}\left(\left\|\left(u^{m}-v^{m}\right) u_{x}^{2}\right\|_{H^{-1}}+\left\|\left(u^{m}-v^{m}\right)_{x} u_{x}^{2}\right\|_{H^{-2}}+c\|w\|_{L^{2}}\right) \\
& \leq c\|w\|_{L^{2}}\left(\left\|u_{x}\right\|_{L^{\infty}}^{2}\|w\|_{L^{2}} \sum_{j=0}^{m-1}\|u\|_{L^{\infty}}^{j}\|v\|_{L^{\infty}}^{m-1-j}+c\|w\|_{L^{2}}\right) \\
& \leq c\|w\|_{L^{2}}^{2} .
\end{aligned}
$$

Using (32)-(34), we get

$$
\frac{1}{2} \frac{d}{d t} \int_{R} w^{2} d x \leq c\|w\|_{L^{2}}^{2}
$$

Applying $w(0)=0$ results in $\|w\|_{L^{2}}^{2}=0$. Consequently, we know that the global weak solution is unique.

\section{Acknowledgment}

This work is supported by the Fundamental Research Funds for the Central Universities (JBK120504). 


\section{References}

[1] R. Camassa and D. D. Holm, "An integrable shallow water equation with peaked solitons," Physical Review Letters, vol. 71, no. 11, pp. 1661-1664, 1993.

[2] R. S. Johnson, "Camassa-Holm, Korteweg-de Vries and related models for water waves," Journal of Fluid Mechanics, vol. 455, no. 1, pp. 63-82, 2002.

[3] R. S. Johnson, "On solutions of the Camassa-Holm equation," Proceedings of the Royal Society A, vol. 459, no. 2035, pp. 1687-1708, 2003.

[4] A. Fokas and B. Fuchssteiner, "Symplectic structures, their Bäcklund transformations and hereditary symmetries," Physica D, vol. 4, no. 1, pp. 47-66, 1981.

[5] A. Constantin and D. Lannes, "The hydrodynamical relevance of the Camassa-Holm and Degasperis-Procesi equations," Archive for Rational Mechanics and Analysis, vol. 192, no. 1, pp. 165-186, 2009.

[6] A. Constantin, "On the scattering problem for the CamassaHolm equation," Proceedings of the Royal Society A, vol. 457, no. 2008, pp. 953-970, 2001.

[7] J. Lenells, "Conservation laws of the Camassa-Holm equation," Journal of Physics A, vol. 38, no. 4, pp. 869-880, 2005.

[8] H. P. McKean, "Fredholm determinants and the Camassa-Holm hierarchy," Communications on Pure and Applied Mathematics, vol. 56, no. 5, pp. 638-680, 2003.

[9] A. Constantin and J. Escher, "Global existence and blow-up for a shallow water equation," Annali della Scuola Normale Superiore di Pisa, vol. 26, no. 2, pp. 303-328, 1998.

[10] A. Constantin, "Existence of permanent and breaking waves for a shallow water equation: a geometric approach," Annales de l'Institut Fourier, vol. 50, no. 2, pp. 321-362, 2000.

[11] A. Constantin, "On the inverse spectral problem for the Camassa-Holm equation," Journal of Functional Analysis, vol. 155, no. 2, pp. 352-363, 1998.

[12] A. Constantin and J. Escher, "Global weak solutions for a shallow water equation," Indiana University Mathematics Journal, vol. 47, no. 4, pp. 1527-1545, 1998.

[13] A. Constantin and B. Kolev, "Geodesic flow on the diffeomorphism group of the circle," Commentarii Mathematici Helvetici, vol. 78, no. 4, pp. 787-804, 2003.

[14] A. Constantin, T. Kappeler, B. Kolev, and P. Topalov, "On geodesic exponential maps of the Virasoro group," Annals of Global Analysis and Geometry, vol. 31, no. 2, pp. 155-180, 2007.

[15] S. Y. Lai and Y. H. Wu, "The local well-posedness and existence of weak solutions for a generalized Camassa-Holm equation," Journal of Differential Equations, vol. 248, no. 8, pp. 2038-2063, 2010.

[16] S. Y. Lai and Y. H. Wu, "A model containing both the Camassa-Holm and Degasperis-Procesi equations," Journal of Mathematical Analysis and Applications, vol. 374, no. 2, pp. 458-469, 2011.

[17] S. Y. Lai and Y. H. Wu, "Existence of weak solutions in lower order Sobolev space for a Camassa-Holm-type equation," Journal of Physics A, vol. 43, no. 9, Article ID 095205, 13 pages, 2010.

[18] A. Bressan and A. Constantin, "Global conservative solutions of the Camassa-Holm equation," Archive for Rational Mechanics and Analysis, vol. 183, no. 2, pp. 215-239, 2007.

[19] A. Bressan and A. Constantin, "Global dissipative solutions of the Camassa-Holm equation," Analysis and Applications, vol. 5, no. 1, pp. 1-27, 2007.
[20] N. Li, S. Y. Lai, S. Li, and M. Wu, "The local and global existence of solutions for a generalized Camassa-Holm equation," Abstract and Applied Analysis, vol. 2012, Article ID 532369, 26 pages, 2012.

[21] B. Kolev, "Poisson brackets in hydrodynamics," Discrete and Continuous Dynamical Systems A, vol. 19, no. 3, pp. 555-574, 2007. 


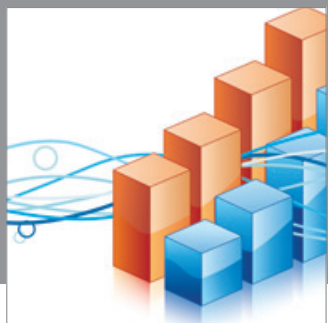

Advances in

Operations Research

mansans

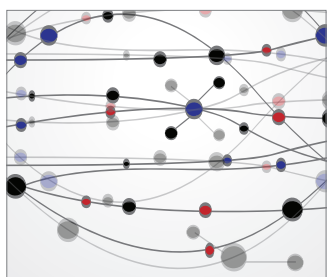

The Scientific World Journal
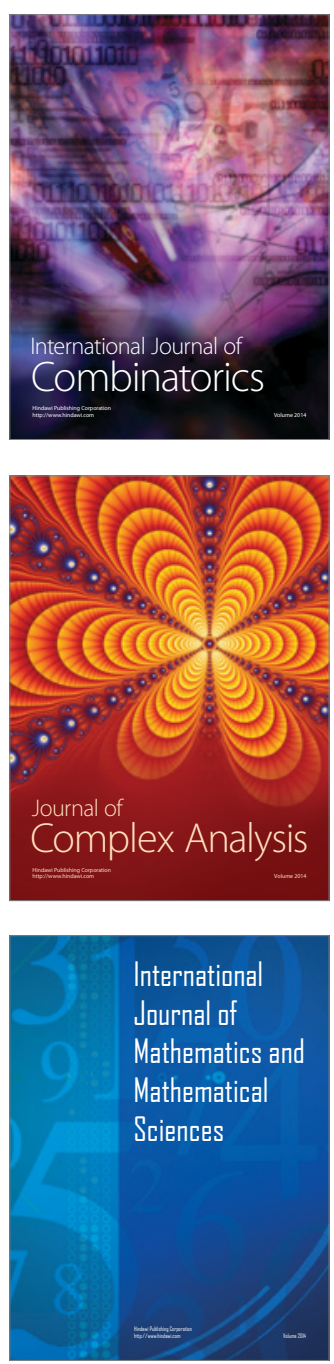
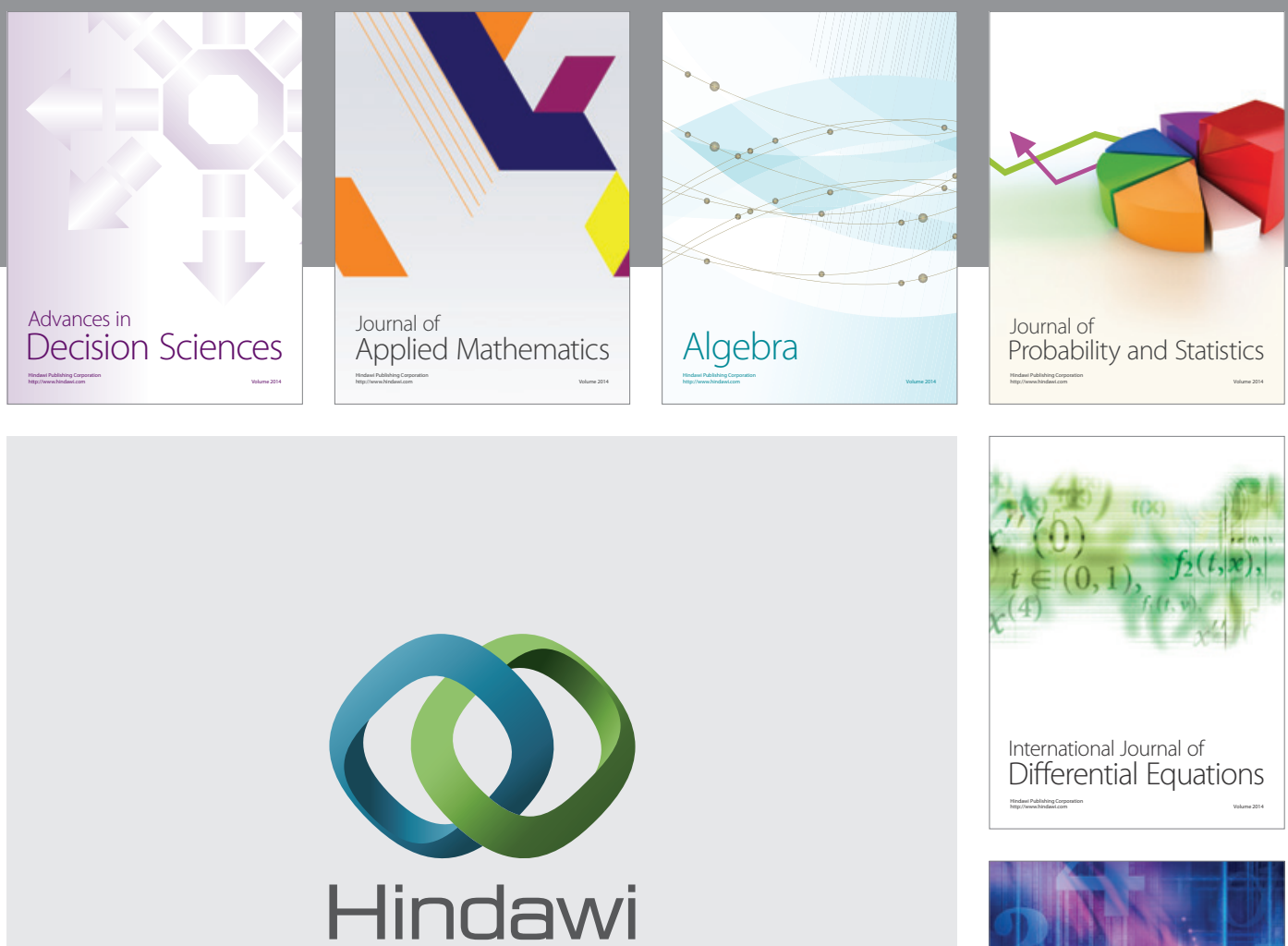

Submit your manuscripts at http://www.hindawi.com
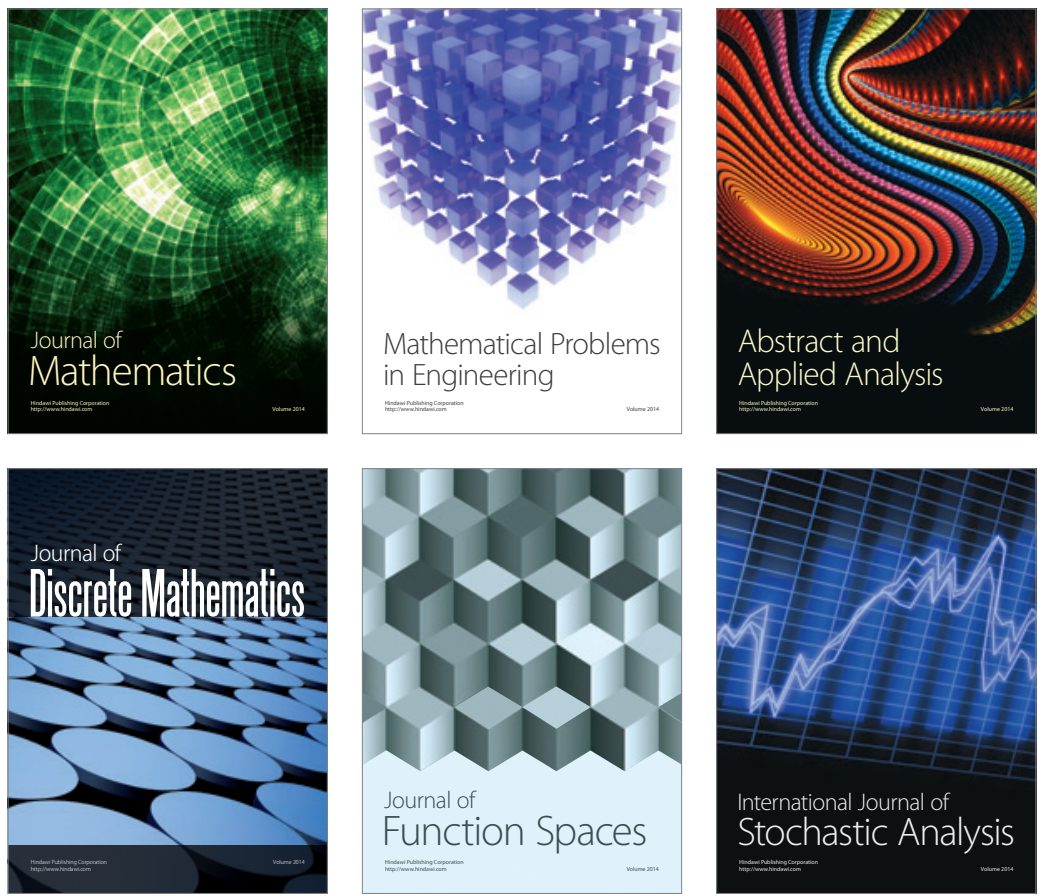

Journal of

Function Spaces

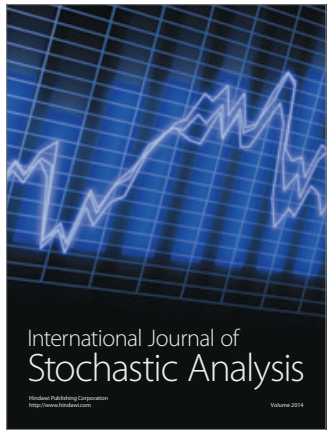

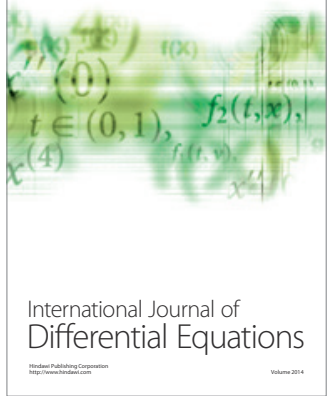
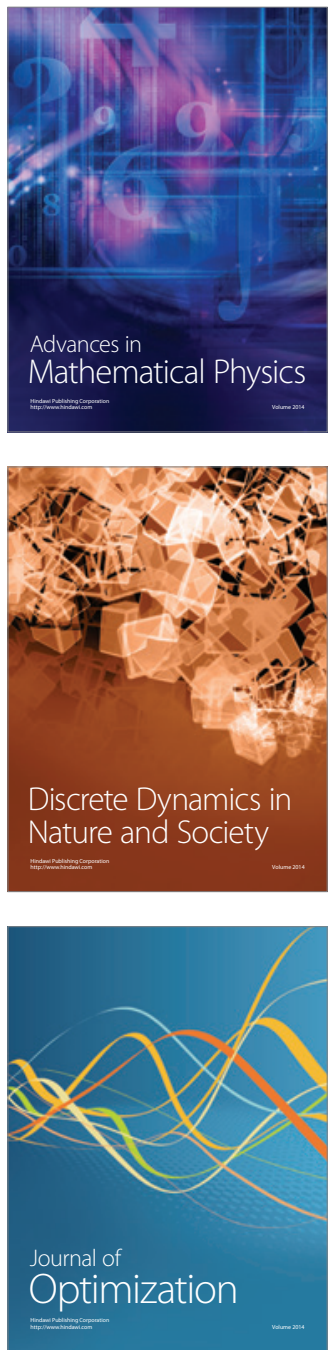